\title{
Markerless Augmented Reality for Robotic Helicoptor Applications
}

\author{
Ian Yen-Hung Chen ${ }^{1}$, Bruce MacDonald ${ }^{1}$, and Burkhard Wünsche ${ }^{2}$ \\ ${ }^{1}$ Dept. of Electrical and Computer Engineering, \\ University of Auckland, New Zealand \\ $\{$ i.chen, b.macdonald\}@auckland.ac.nz \\ ${ }^{2}$ Dept. of Computer Science, University of Auckland, New Zealand \\ burkhard@cs.auckland.ac.nz
}

\begin{abstract}
The objective of this research is to apply markerless Augmented Reality (AR) techniques to aid in the visualisation of robotic helicopter related tasks. Conventional robotic AR applications work well with markers in prepared environments but are infeasible in outdoor settings. In this paper, we present preliminary results from a real time markerless AR system for tracking natural features in an agricultural scene. By constructing a virtual marker under a known initial configuration of the robotic helicopter, camera and the ground plane, the camera pose can be continuously tracked using the natural features from the image sequence to perform augmentation of virtual objects. The experiments are simulated on a mock-up model of an agricultural farm and the results show that the current AR system is capable of tracking the camera pose accurately for translational motions and roll rotations. Future work includes reducing jitter in the virtual marker vertices to improve camera pose estimation accuracy for pitch and yaw rotations, and implementing feature recovery algorithms.
\end{abstract}

\section{Introduction}

The term Augmented Reality (AR) refers to the integration of virtual elements onto a view of a real world environment. This is achieved by superimposing virtual or synthetic computer generated information onto the live video of the real world captured from cameras. While the augmentation is being performed, the synthetic information will be displayed in real time on AR display devices such as monitors, projection screens, or head mounted displays (HMD) [1]. There are a growing number of applications that aim to enhance the AR experience with other sensory cues such as haptic feedback or spatial directional audio [2]3]4. AR can be found in many application domains, such as entertainment [5], archaeology [6], touring [7, robotics [8, medical uses 9], military training [10, and more [11]12.

One of the main challenges in producing effective AR applications lies in the accurate registration of synthetic information in the real world, that is, the alignment between the virtual and the real [13. Small errors in the alignment

G. Sommer and R. Klette (Eds.): RobVis 2008, LNCS 4931, pp. 125-138 2008.

(C) Springer-Verlag Berlin Heidelberg 2008 
are easily perceptible to the human eye, and therefore, fast, precise and robust tracking techniques are essential for computing an accurate position and orientation of the camera 1314. Marker-based tracking methods can be a fast, low cost solution to many AR applications. By tracking markers of known size in the environment, the marker vertices and direction information can be extracted to determine the camera pose and produce accurate registration results [15. However, there are a number of drawbacks associated with artificial markers, especially for outdoor AR applications in unprepared environments. Partial occlusion of the markers or direct exposure to strong lighting conditions would cause tracking to produce erroneous registration results. Moreover, the camera will be constrained to look only at regions where the markers are in view, hence limiting the users' working space 13 .

The use of natural features in the scene for tracking is more desirable. No a priori information need be known and modifications to the environment are avoided. You et al. [16 propose a hybrid tracking technique that predicts motion estimates using inertial sensors. The accumulated drift errors are then corrected by vision at regular time intervals. Cornelis et al. [17] develop an AR system from uncalibrated video sequences, based on motion and structure recovery techniques and preprocessing of images to specify key frames for computing the fundamental matrix that relates corresponding points in stereo images. Wu et al. 18 also make use of fundamental matrices at key frames to derive the camera pose, and simplify the registration problem by placing control points of known positions in the scene to resolve scale ambiguity. Yuan et al. 19] propose robust accurate registration in real time by using a projective reconstruction approach to recover camera pose from four planar points.

The application of AR technology to the field of robotics visualisation has several benefits. It helps to visualise information, such as robot sensory data or historic paths, in context with the physical world 8 . A number of robotic AR applications exist which aim to improve the effectiveness of the robot software development process by providing real time visual feedback to robot developers. Pettersen et al. [20] use marker-based AR to visualise the results in robot painting tasks and obtain instant feedback. Stilman et al. 21] use AR for debugging vision and motion planning algorithms with humanoid robots, using motion capture systems and markers. Collett and MacDonald 8 also develop a marker-based AR visualisation tool to help robot developers debug robotic software by highlighting the inconsistencies between the robot's world view and the real world. Moreover, $\mathrm{AR}$ is able to help users monitor robots and the environment from remote areas. Sugimoto et al. 22 use AR to aid teleoperation of robots, by presenting an exocentric view of the robot based on images taken from an egocentric camera. Brujic-Okreti et al. 23] assist users in remote-control of robotic vehicles by displaying AR visualisations of the vehicle and the environment using digital elevation models and simulated GPS readings.

The research proposed in this paper applies natural feature tracking techniques to the development of AR systems for visualisation in robotic helicopter tasks. In contrast to existing robotic AR applications, the proposed method over- 
comes the disadvantages of marker-based tracking techniques and scales robotic AR applications to operate in outdoor environments. It uses a simpler registration process than existing natural feature tracking AR applications. This is made possible by taking advantage of robotic actuator capabilities at initialisation to position the camera at an orientation perpendicular to the target plane. Under this condition, only two parameters are required to define the planar region, the centre point and the size of the virtual object. They are represented by a virtual marker, on which the augmentation will take place. Once the AR system is initiated, the robotic helicopter is free to move and change its orientation, and the virtual elements will remain aligned with the real world.

Section 2 describes the problem to be solved. Section 3 presents our solution to markerless tracking and registration. Section 4 summarises our overall AR system design. Section 5 gives results for a simulation. Section 6 discusses the future improvements and Section 7 concludes the paper.

\section{Problem Description}

Our focus is on using AR visualisation to assist robot developers when they are creating robotic helicopter applications for the agricultural industry. Common vision-related robotic helicopter tasks in agriculture include remote sensing, spraying, and monitoring of crops and livestock for better farm management [24|25|26 27. The tasks typically use onboard cameras to observe the agricultural objects in the field. The ultimate goal is a framework for applying AR techniques in the agricultural environment with the use of camera images captured from a robotic helicopter. This will involve identifying the important requirements and evaluating the algorithms developed as a result of them. It is also necessary to resolve the registration problem to produce accurate alignment of the virtual objects and the real world, in response to the motions of the robotic helicopter and a pan tilt camera.

The objective is to track the pose of an intrinsically calibrated camera to facilitate the registration of a virtual object under a known initial configuration of the robotic helicopter, camera and the ground plane. It is advantageous to make use of the actuators on the robotic helicopter platform to adjust the orientation of the camera at the initial start-up of the system. The camera is oriented directly downwards at the agriculture field before commencing AR visualisation. This can be achieved by issuing a command to robotic actuator devices such as pan-tilt camera holders to alter to the desired orientation. The resulting configuration of the camera mounted on the robotic helicopter and the environment is illustrated in Fig. 1. It is also assumed that there are sensors onboard which are able to give an accurate estimate of the height of the robotic helicopter from the ground to initialise the registration process. The problem then becomes the tracking of the camera position and orientation under this initial configuration and keeping the virtual objects aligned with the real world while the robotic helicopter moves or changes its angle of view. This is a simplified version of a real world problem. The helicopter would not always be exactly parallel to the ground plane and future improvements must be implemented to address this issue. 


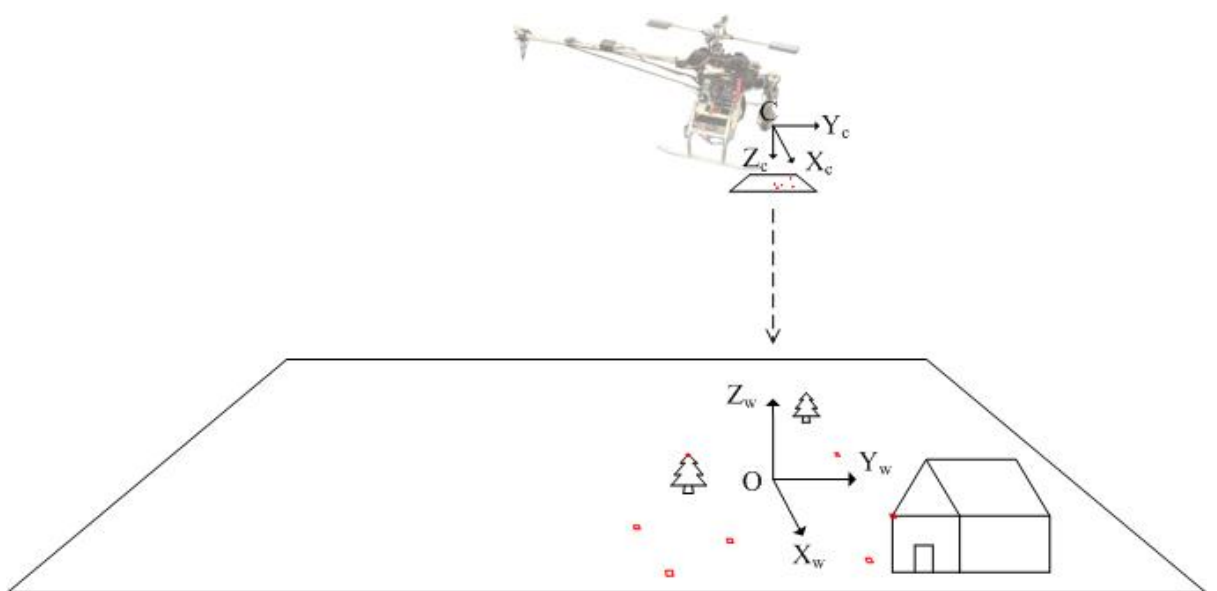

Fig. 1. Initial configuration of the camera and the environment with the camera oriented at an angle perpendicular to the ground plane. $\mathrm{X}_{\mathrm{C}}, \mathrm{Y}_{\mathrm{C}}$ and $\mathrm{Z}_{\mathrm{C}}$ denote the axes of the camera coordinate system, and $\mathrm{X}_{\mathrm{W}}, \mathrm{Y}_{\mathrm{W}}$ and $\mathrm{Z}_{\mathrm{W}}$ form the world coordinate system

The unique behaviour of robotic helicopters compared to other fixed wing unmanned aerial vehicles is that they have the ability to hover over the agricultural objects and perform both rotational and translational motion with six degrees of freedom as shown in Fig. 2. A yaw rotation in the helicopter will result in a roll of the camera, and similarly, a roll rotation of the helicopter results in a yaw of the camera. The proposed system will apply natural feature tracking techniques to derive the camera pose for accurate registration of virtual objects while dealing with the different motions of the robotic helicopter.

\section{Markerless Tracking and Registration}

\subsection{Augmented Reality Initialisation}

The Kanade-Lucas-Tomasi (KLT) feature tracker 28|29] has been chosen for real time tracking of natural features in the scene. The KLT algorithm has been shown to achieve great performance in terms of speed and accuracy compared to other feature extracters for small camera motions and viewpoints between consecutive images [30. In addition, it provides its own feature matching algorithm for tracking feature points in image pairs based on a model of affine image changes. The KLT feature tracker has been successfully applied in several outdoor AR systems [31/32 33/34].

A number of initial steps must be taken before proceeding to the augmentation of virtual objects. First, two reference images of the scene are required. The KLT tracking algorithm is applied to identify, select, and track natural features in these image frames. The features that are tracked between these two images are used to compute the Fundamental Matrix $F$ using the Normalised Eight 


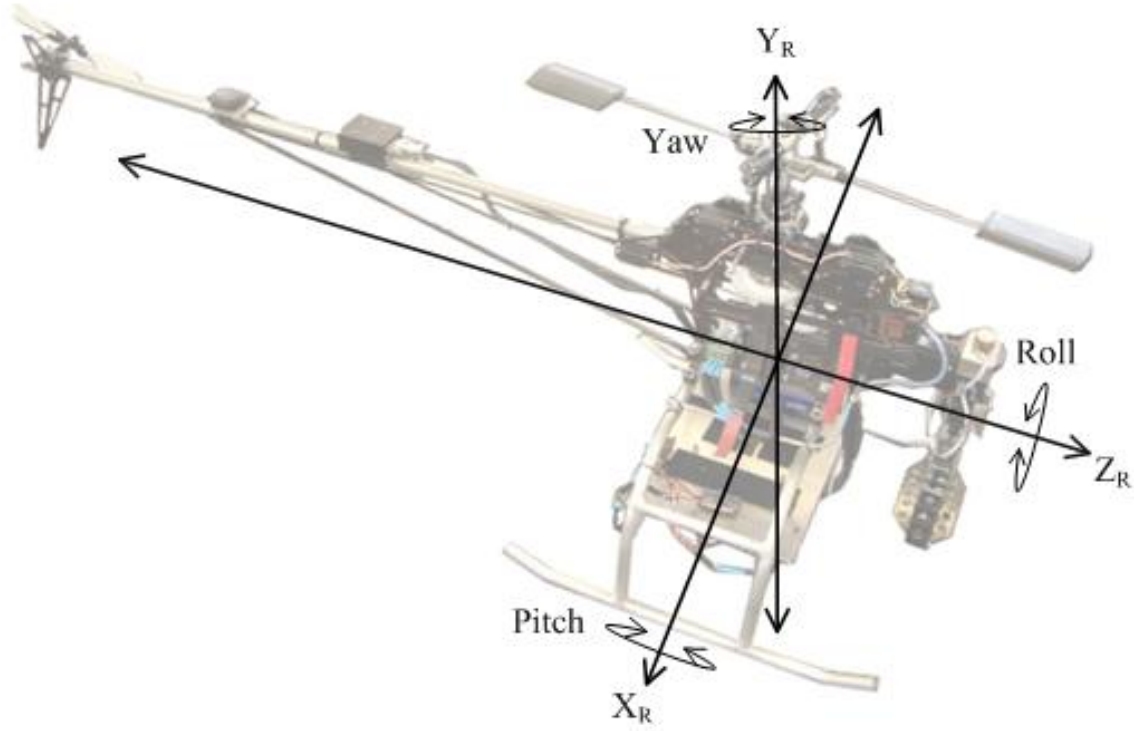

Fig. 2. Robotic helicopter with 6 degrees of freedom. Translation in X,Y and Z direction in the local robot coordinate system plus pitch, yaw and roll rotations.

Point algorithm proposed by Hartley 35, which has been shown to improve the estimation of $F$ when working with noisy inputs. The fundamental matrix is essentially an algebraic representation of epipolar geometry in which the two corresponding matching image points, $x^{\prime}$ and $x$, are related by the equation:

$$
x^{\prime T} F x=0
$$

In this case, the pair of matching images points are the natural features tracked by the KLT feature tracker in the two reference images. From $F$, a pair of camera projection matrices, $P$ and $P^{\prime}$, can be retrieved in the form of:

$$
P=[I \mid O], P^{\prime}=\left[\left[e^{\prime}\right]_{\times} F \mid e^{\prime}\right]
$$

where $\mathrm{I}$ is the $3 \times 3$ identity matrix, $\mathrm{O}$ is a null 3 -vector, and $e^{\prime}$ is the epipole of the second reference image. The detailed mathematical derivations and proofs can be found in [36]. The pair of projection matrices, $P$ and $P^{\prime}$, establishes a projective space where the $3 \mathrm{D}$ coordinates $X$ of the tracked image point matches can be constructed using a linear method from the equations:

$$
x=P X, x^{\prime}=P^{\prime} X
$$

The 3D coordinates $X$ will remain constant throughout the execution of the system, assuming that (a) the major portion of the environment remains static 
and (b) there are smoothing techniques available to eliminate outliers from the set of matching image points, such as wrongly tracked features or moving objects in the real world. It is also important to note that the two reference images should be captured from points wide apart to reduce the uncertainties in the reconstructed 3D points 17 .

To indicate the position where the virtual object will be overlaid, the user needs only to specify a point on the second reference image which represents the centre of virtual objection registration. Since the camera on the robotic helicopter is facing in a direction perpendicular to the ground plane, a square-shaped virtual marker can be rapidly constructed with four virtual vertices equally spaced from the specified point in the 2D image plane. Their corresponding 3D points can be constructed using $P^{\prime}$. The method is similar to the projective reconstruction technique proposed by Yuan et al. [19, but in comparison, the registration process is simplified by starting with the camera orientation suggested in this research. It prevents users from the error-prone process of manually specifying the four coplanar points in the 2D image plane as viewed by a camera under perspective transformation, since small errors in the input will lead to inaccurate registration results. The four vertices of the virtual marker will play an important role, as will be seen later, for estimating the camera pose in consecutive image frames.

\subsection{Camera Tracking}

As the helicopter moves, the position and orientation of the virtual camera should also be updated accordingly such that the virtual objects remain aligned with the real world. There must be a new projection matrix associated with every consecutive frame. The problem can be solved by employing triangulation techniques with matching $3 \mathrm{D}$ to $2 \mathrm{D}$ correspondences. Given that the KLT feature tracker will be able to provide the $2 \mathrm{D}$ image coordinates of the natural features tracked at each image frame, and the reconstructed 3D points in the unique projection space remain unchanged, the projection matrix $P_{k}$ for the $k^{t h}$ image can be calculated using the Gold Standard algorithm [36] with at least six 3D to $2 \mathrm{D}$ point correspondences. To summarise, the $2 \mathrm{D}$ image points and $3 \mathrm{D}$ reconstructed points are first normalised, and the Direct Linear Transformation (DLT) algorithm is then applied to compute an initial estimate of $P_{k}$. To obtain a more robust result, the iterative Levenberg-Marquardt algorithm is used to minimise the geometric error:

$$
\sum_{i} d\left(x_{i}, P X_{i}\right)^{2}
$$

where $x_{i}$ and $X_{i}$ are in normalised form and $P$ is parameterised with the twelve elements from the initial linear estimate of $P_{k}$. Denormalisation is then performed to obtain the final resulting $P_{k}$. 
By obtaining the projection matrix for every consecutive image frame in the video sequence, the $2 \mathrm{D}$ image coordinates of the virtual vertices can be reprojected using Equation (3) from their corresponding 3D points in the unique projection space. Assuming that the intrinsic parameters of the camera are known in advance and stay constant throughout the entire augmentation, the camera pose for the $k^{\text {th }}$ image frame can be computed by using the reprojected image coordinates of the virtual marker vertices as input to the Robust Pose Estimation Algorithm for Planar Targets 37/38. The rotation and translation motion of the camera can be derived relative to the virtual marker's pose.

The 3D virtual object will be registered at the origin of the world coordinate system sitting on the XY plane and the camera will be initially placed along the positive $\mathrm{Z}$ axis looking down at the virtual object as shown in Fig. 1, The appropriate rotation and translation motion derived at the $k^{\text {th }}$ image frame will be applied to the virtual camera so the virtual objects remains aligned with the real world when the position and orientation of the real camera changes.

\section{System Design}

In order to create useful AR applications, the quality of the graphics embedded in the AR system is important. A dedicated open source $3 \mathrm{D}$ graphics rendering engine, OGRE 3D [39], has been chosen. It has a large community support and provides advanced graphics rendering functionalities including a material shader, scripted animations and other special effects. The use of the OGRE 3D graphics rendering engine allows the creation of high quality computer graphics in a short period of time. Moreover, for future considerations, it has the capability to integrate physics and networking libraries to construct a more powerful AR or Mixed Reality (MR) system.

The interface to robot sensors and actuators is made possible by Player/Stage 40] which is a popular open source software tool widely used in the research and development of robot and sensor applications. The Player server will be run on the robot platform and provides developers an interface for controlling the underlying hardware over the IP network. To connect to the Player server, a Player plug-in for OGRE 3D is created. It is essentially a Player client that acquires raw camera image data from the camera mounted on the robot platform. The camera images are compressed on the Player server first before being streamed across the network and decompressed on the client side to optimize bandwidth efficiency. The markerless AR system is implemented by utilising this plug-in and rendering the acquired camera images as dynamic textures on the background in the OGRE 3D window in colour, but they are converted to a gray scale format before feeding into the KLT feature tracker. The virtual objects to be rendered over the camera images can be created in other modeling tools and then loaded into OGRE 3D. Fig. 3 shows the overall system design and the data flow between the different components. 


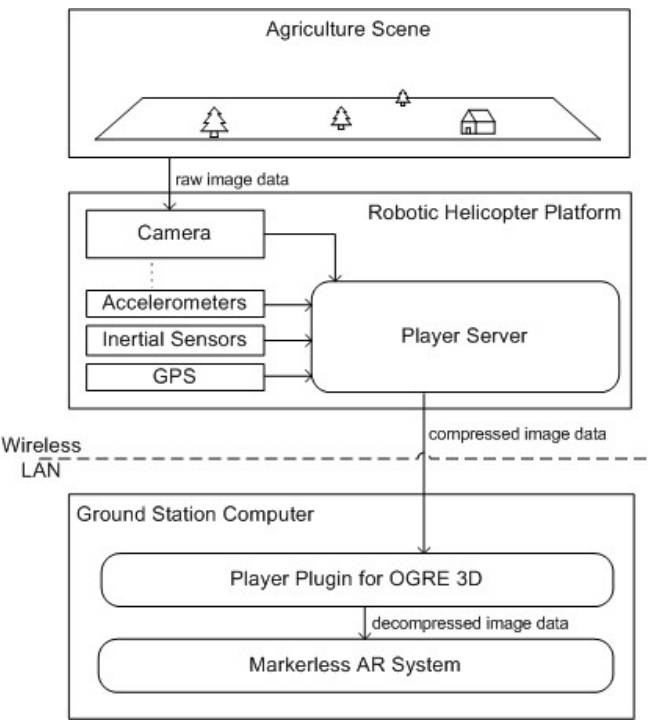

Fig. 3. The overall system design and data flow

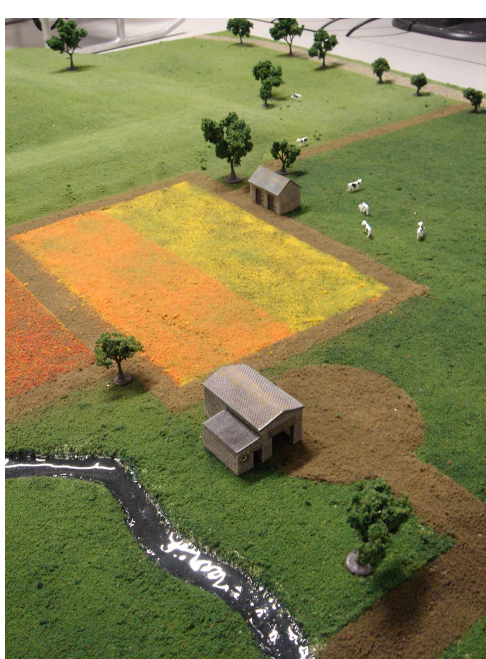

Fig. 4. Mock-up model of an agricultural farm

\section{Experimental Results from Simulation}

As robotic helicopter applications need to deal with noisy images, changes in illuminations, and different outdoor scene features, we have designed an outdoor scene which simulates an agricultural environment. The experiments were performed over a mock-up model of an agricultural farm shown in Fig. 4. The model is at the scale 1:200 with a base size of $\mathrm{A} 0$ and a maximum height of 10 centimeters. The model was built in the University of Auckland Robotics Laboratory, for simulating various robotic helicopter tasks, particularly robot vision related projects, before venturing into real world experimentation. A variety of realistic agricultural objects are added and lighting equipment is available to simulate a real life farm under different time-of-day conditions. There are other issues regarding this simulation approach which need to be considered in the future, such as the missing effect of shadows from moving clouds, simulating different weather conditions, and the realism of objects and illuminations. However, it provides us an insight to the characteristics of an outdoor environment and the major problems that could arise when operating robotic systems outdoors, which include working with different texture contents in an agricultural scene, repetitive scene patterns, the variety of shapes of agricultural objects, and motioning in an unstructured environment.

First, two well-spaced reference images of the scene were captured and the natural features were tracked using the KLT feature tracker. A virtual barn was augmented starting at the centre of the image. Then the camera underwent a series of motions. 


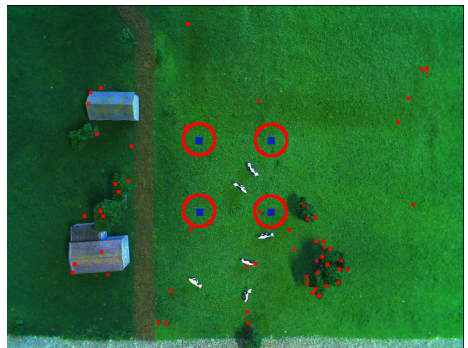

(a)

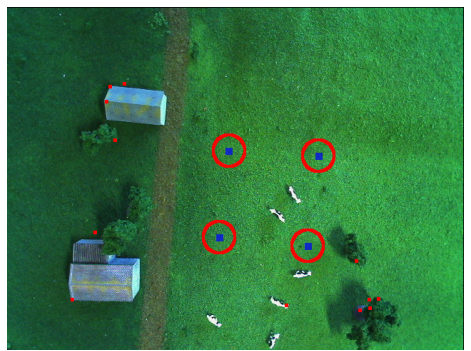

(c)

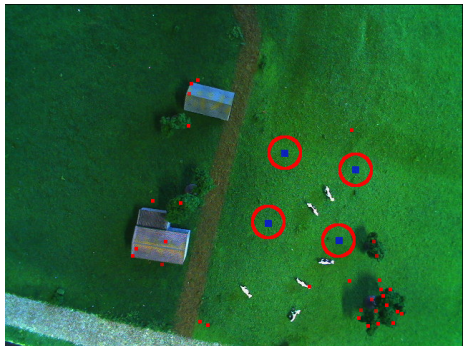

(b)

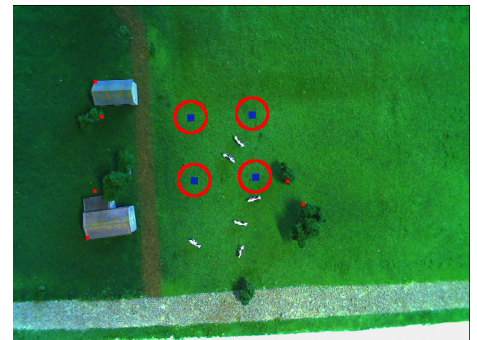

(d)

Fig. 5. The resulting reprojections of the virtual marker vertices after undergoing a series of camera motions. The marker vertices are blue squares, highlighted in these images by red circles.

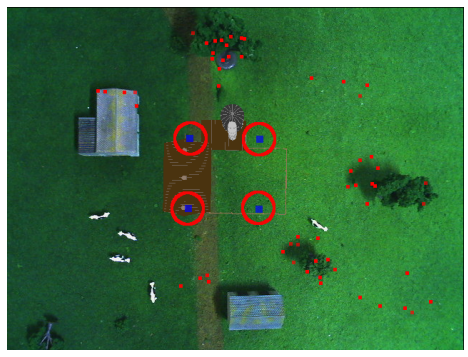

(a)

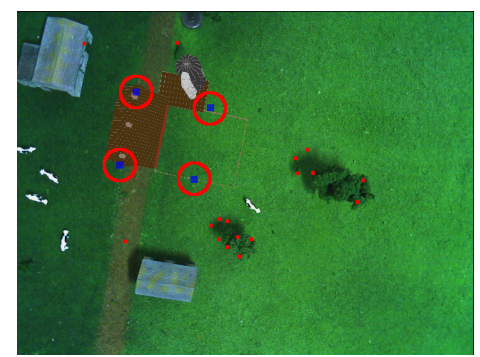

(c)

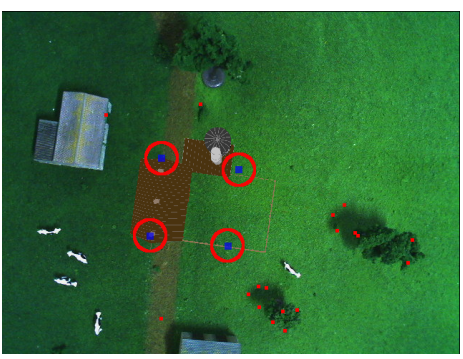

(b)

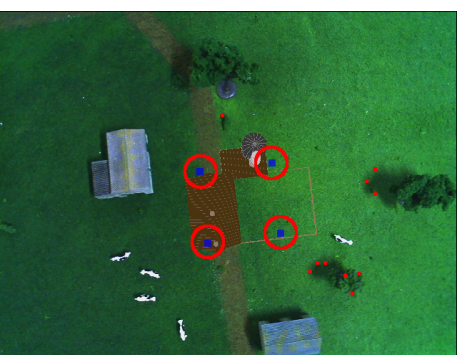

(d)

Fig. 6. Augmentation of a virtual barn on the virtual marker. The marker vertices are blue squares, highlighted in these images by red circles. 
The system was deployed in Linux on a $2.4 \mathrm{GHz}$ Intel(R) Core(TM)2 Quad CPU with 1GB of RAM and a NVIDIA Quadro FX 3450/4000 SDI graphics card. A Logitech Quickcam Fusion camera was used to capture the images of size $640 \times 480$. The frame rate of the markerless AR system remained at approximately 4-5 frames per second for the entire augmentation.

Fig. 5 shows the virtual marker vertices being reprojected in different frames. The red rectangular blobs represent the natural features tracked by the KLT feature tracker and the four blue rectangular blobs are the vertices that form a square-shaped virtual marker. Fig. 5(a), 5(b) show the result of the virtual marker vertices when moving the camera in $\mathrm{X}$ and $\mathrm{Y}$ directions while undergoing some rotation, and $5(\mathrm{c}), 5(\mathrm{~d})$ show the effect of moving the camera along the $\mathrm{Z}$ axis. Fig. 6 shows the result of placing the virtual barn in the origin of the world coordinate system while the virtual camera position and orientation are being updated using the Robust Pose Estimation algorithm.

An experiment was conducted to measure the residual error between the predicted camera position and the actual world position for translational motions. The camera was placed at $40 \mathrm{~cm}$ from the ground plane and translated in its local $\mathrm{X}, \mathrm{Y}$ and $\mathrm{Z}$ directions. The virtual object was augmented at the centre of the image as before and the residual error was recorded at increments of $1 \mathrm{~cm}$ for an overall translation of $15 \mathrm{~cm}$.

The experiment was repeated 8 times and the error after the full translation was found to be an average of $1.77 \mathrm{~cm}$ in the $\mathrm{X}$ direction, $1.40 \mathrm{~cm}$ in the $\mathrm{Y}$ direction, and $1.25 \mathrm{~cm}$ in the $\mathrm{Z}$ direction. Equivalently, the expected error when operated on a real farm would be approximately $3.54 \mathrm{~m}, 2.80 \mathrm{~m}, 2.50 \mathrm{~m}$ in the $\mathrm{X}$, $\mathrm{Y}$, and $\mathrm{Z}$ direction, respectively. The error was observed to increase incrementally as the result of losing the tracked natural features, mainly when they moved out of the camera view. Consequently, the registration error became obvious to the human eye when the virtual object was situated near the edges of the image plane.

\section{Discussions and Future Work}

The AR system was able to correctly update the camera pose as shown in Section 5. The augmentation of virtual objects was accurate for translations of the camera and the errors were minor. The noise in the tracked natural features had a larger impact on pitch and yaw rotations compared to roll rotations. The reason is that the virtual marker will no longer be square-shaped in the image image plane due to perspective transformation and minor pixel errors in the reprojections of virtual marker vertices will cause the Robust Pose Estimation algorithm to think that the camera is at a largely different position and orientation. Ultimately, the accuracy of the registration is highly dependent on the natural feature being tracked. There will be errors in the virtual object registration due to jitter in the virtual marker vertices, which would cause the Robust Pose Estimation algorithm to produce unstable results between image frames. 
The instability in the virtual maker vertices will have a more significant effect on the computed camera rotations. To solve the problem, a more robust algorithm needs to be applied to filter the noise and wrongly tracked natural features. Other feature tracking algorithms could also be considered which would produce higher distinctive features to obtain better results, but the trade-off could be a higher computational cost. This needs to be further investigated.

There are also a number of limitations in the current AR system if it is to be deployed for robotic helicopter flight. An automated feature recovering algorithm is necessary. As the robotic helicopter is a noisy and unstable platform, there will be situations where the helicopter will undergo sudden large motions. In this case, the natural features would be lost and augmentation would fail. Relying on vision techniques alone without artificial landmarks or external inputs in the environment presents difficulties to recover the camera pose quickly online. Therefore, a hybrid approach incorporating onboard sensors will be considered in the future, to provide rough estimates of the camera pose when features are lost. Then vision techniques can be used to refine these estimates to resume augmentation. To overcome the constraint imposed by the initial configuration mentioned in Section 2, the pose of the ground plane also needs to be determined in the initialization stage. Furthermore, to scale the system to operate in large working area, more natural features would need to be tracked outside the scene covered by the reference images. The system should be automated to recover the 3D structure information of the scene from the incoming camera images as the robotic helicopter travels between different areas of the agricultural land. Methods to achieve this include capturing more reference images during the preliminary offline process [41, or by tracking a virtual 3D plane from the computation of plane homographies over the image sequences [42].

\section{Conclusions}

This research investigates the essential tracking requirements for applying the augmented reality visualisation technique in robotic helicopter applications. A markerless AR system based on natural feature tracking is presented. We have successfully tracked the camera pose by introducing a virtual marker into the scene, which achieves good results subject to the proposed initial configuration of the camera, robotic helicopter, and the environment. We have developed a simulation using a mock-up model of an agriculture farm for testing the performance of the markerless AR system. From the experiments we can conclude that there are a number of improvements which need to be taken in the future before deploying the system in actual flight. The jittering motion of the virtual marker vertices must be reduced for better camera pose estimation, and most importantly, a feature recovery algorithm is crucial to scale the markerless AR system to operate outdoors on the robotic helicopter. 


\section{References}

1. Milgram, P., Kishino, F.: A taxonomy of mixed reality visual display. IEICE Transactions on Information Systems E77-D, 1321-1329 (1994)

2. Cheok, A.D., et al.: Capture the flag: a multiplayer online game for phone users. In: Proceedings of the Ninth IEEE International Symposium on Wearable Computers, 2005, October 18-21, 2005, pp. 222-223 (2005)

3. Hughes, C.E., et al.: Mixed reality in education, entertainment, and training. IEEE Computer Graphics and Applications 25(6), 24-30 (2005)

4. Sandor, C., et al.: Immersive mixed-reality configuration of hybrid user interfaces. In: Proceedings of the Fourth IEEE and ACM International Symposium on Mixed and Augmented Reality, 2005, October 5-8, 2005, pp. 110-113 (2005)

5. Thomas, B., et al.: Arquake: An outdoor/indoor augmented reality first person application. In: The Fourth International Symposium on Wearable Computers, 2000, October 16-17, 2000, pp. 139-146 (2000)

6. Benko, H., Ishak, E.W., Feiner, S.: Collaborative mixed reality visualization of an archaeological excavation. In: Third IEEE and ACM International Symposium on Mixed and Augmented Reality, 2004. ISMAR 2004, November 2-5, 2004, pp. 132-140 (2004)

7. Vlahakis, V., et al.: Personalized augmented reality touring of archaeological sites with wearable and mobile computers. In: Horrocks, I., Hendler, J. (eds.) ISWC 2002. LNCS, vol. 2342, pp. 15-22. Springer, Heidelberg (2002)

8. Collett, T., MacDonald, B.: Augmented reality visualisation for player. In: Proceedings of the 2006 IEEE International Conference on Robotics and Automation, 2006. ICRA 2006, pp. 3954-3959 (2006)

9. Rosenthal, M., et al.: Augmented reality guidance for needle biopsies: A randomized, controlled trial in phantoms. In: Niessen, W.J., Viergever, M.A. (eds.) MICCAI 2001. LNCS, vol. 2208, pp. 240-248. Springer, Heidelberg (2001)

10. Livingston, M.A., et al.: An augmented reality system for military operations in urban terrain. In: Proceedings of the Interservice / Industry Training, Simulation, \& Education Conference (I/ITSEC 2002), Orlando, December 2-5 (2002)

11. Azuma, R.T.: A survey of augmented reality. Presence: Teleoperators and Virtual Environments 6(4), 355-385 (1997)

12. Azuma, R., et al.: Recent advances in augmented reality. IEEE Computer Graphics and Applications 21(6), 34-47 (2001)

13. Azuma, R.T.: 21. In: Mixed Reality: Merging Real and Virtual Worlds, pp. 379390. Springer, Heidelberg (1999)

14. Bimber, O., Raskar, R.: Spatial Augmented Reality: Merging Real and Virtual Worlds. A.K. Peters, Ltd., Natick, MA, USA (2005)

15. Kato, H., Billinghurst, M.: Marker tracking and hmd calibration for a video-based augmented reality conferencing system. In: Proceedings of the 2nd IEEE and ACM International Workshop on Augmented Reality, 1999 (IWAR 1999), pp. 85-94 (1999)

16. You, S., Neumann, U., Azuma, R.: Orientation tracking for outdoor augmented reality registration. IEEE Computer Graphics and Applications 19(6), 36-42 (1999)

17. Cornelis, K., et al.: Augmented reality using uncalibrated video sequences. In: Pollefeys, M., et al. (eds.) SMILE 2000. LNCS, vol. 2018, pp. 144-160. Springer, Heidelberg (2001)

18. Wu, J., Yuan, Z., Chen, J.: A registration method of ar based on fundamental matrix. In: 2005 IEEE International Conference Mechatronics and Automation, vol. 3, pp. 1180-1184 (2005) 
19. Yuan, M., Ong, S., Nee, A.: Registration using natural features for augmented reality systems. IEEE Transactions on Visualization and Computer Graphics 12(4), 569-580 (2006)

20. Pettersen, T., et al.: Augmented reality for programming industrial robots. In: Proceedings of the Second IEEE and ACM International Symposium on Mixed and Augmented Reality, 2003, pp. 319-320 (2003)

21. Stilman, M., et al.: Augmented reality for robot development and experimentation. Technical Report CMU-RI-TR-05-55, Robotics Institute, Carnegie Mellon University, Pittsburgh, PA (November 2005)

22. Sugimoto, M., et al.: Time follower's vision: a teleoperation interface with past images. IEEE Computer Graphics and Applications 25(1), 54-63 (2005)

23. Brujic-Okretic, V., et al.: Remote vehicle manoeuvring using augmented reality. In: International Conference on Visual Information Engineering, 2003. VIE 2003, pp. 186-189 (2003)

24. Sugiura, R., et al.: Field information system using an agricultural helicopter towards precision farming. In: Proceedings of the 2003 IEEE/ASME International Conference on Advanced Intelligent Mechatronics, 2003. AIM 2003, vol. 2, pp. 1073-1078 (2003)

25. Archer, F., et al.: Introduction, overview, and status of the microwave autonomous copter system (macs). In: Proceedings of the 2004 IEEE International Geoscience and Remote Sensing Symposium, 2004. IGARSS 2004, vol. 5, pp. 3574-3576 (2004)

26. Yokobori, J., et al.: Variable management for uniform potato yield using remote sensing images with unmanned helicopter. In: ASAE Annual Meeting (2003)

27. Noguchi, N., Ishii, K., Sugiura, R.: Remote-sensing technology for vegetation monitoring using an unmanned helicopter. In: Biosystems Engineering, pp. 369-379 (2005)

28. Lucas, B.D., Kanade, T.: An iterative image registration technique with an application to stereo vision. In: Proceedings of the 7th International Joint Conference on Artificial Intelligence (IJCAI 1981), April 1981, pp. 674-679 (1981)

29. Shi, J., Tomasi, C.: Good features to track. In: IEEE Computer Society Conference on Computer Vision and Pattern Recognition, 1994. Proceedings CVPR 1994, vol. 1994, pp. 593-600 (1994)

30. Klippenstein, J., Zhang, H.: Quantitative evaluation of feature extractors for visual slam. In: Fourth Canadian Conference on Computer and Robot Vision, 2007. CRV 2007, pp. 157-164 (2007)

31. Yao, A., Calway, A.: Robust estimation of 3-d camera motion for uncalibrated augmented reality. Technical Report CSTR-02-001, Department of Computer Science, University of Bristol (March 2002)

32. Kameda, Y., Takemasa, T., Ohta, Y.: Outdoor see-through vision utilizing surveillance cameras. In: Third IEEE and ACM International Symposium on Mixed and Augmented Reality, 2004. ISMAR 2004, pp. 151-160 (2004)

33. Ong, S.K., Yuan, M.L., Nee, A.Y.C.: Tracking points using projective reconstruction for augmented reality. In: Proceedings of the 3rd international conference on Computer graphics and interactive techniques in Australasia and South East Asia. GRAPHITE 2005, pp. 421-424. ACM Press, New York (2005)

34. Behringer, R., Park, J., Sundareswaran, V.: Model-based visual tracking for outdoor augmented reality applications. In: Proceedings of the International Symposium on Mixed and Augmented Reality, 2002. ISMAR 2002, pp. 277-322 (2002)

35. Hartley, R.: In defense of the eight-point algorithm. IEEE Transactions on Pattern Analysis and Machine Intelligence 19(6), 580-593 (1997) 
36. Hartley, R.I., Zisserman, A.: 8. In: Multiple View Geometry in Computer Vision, 2nd edn., Cambridge University Press, Cambridge (2003)

37. Schweighofer, G., Pinz, A.: Robust pose estimation from a planar target. IEEE Transactions on Pattern Analysis and Machine Intelligence 28(12), 2024-2030 (2006)

38. Wagner, D., Schmalstieg, D.: Artoolkitplus for pose tracking on mobile devices. In: Computer Vision Winter Workshop 2007, February 6-8 (2007)

39. OGRE Ogre 3d: Object-oriented graphics rendering engine, http://www.ogre3d.org

40. Gerkey, B.P., Vaughan, R.T., Howard, A.: The player/stage project: Tools for multi-robot and distributed sensor systems. In: Proceedings of the International Conference on Advanced Robotics (ICAR 2003), June 30-July 3, 2003, pp. 317-323 (2003)

41. Skrypnyk, I., Lowe, D.: Scene modelling, recognition and tracking with invariant image features. In: Third IEEE and ACM International Symposium on Mixed and Augmented Reality, 2004. ISMAR 2004, pp. 110-119 (2004)

42. Lourakis, M., Argyros, A.: Vision-based camera motion recovery for augmented reality. In: CGI 2004. Proceedings of the Computer Graphics International, pp. 569-576. IEEE Computer Society Press, Washington, DC, USA (2004) 